\title{
Imagem Corporal e Comportamentos de Risco para Transtornos Alimentares em Bailarinos Profissionais
}

\author{
Body Image And Risk Behavior for Eating Disorders in Professional \\ Ballet Dancers
}

Lena Guimarães Ribeiro

Gloria Valeria da Veiga

Instituto de Nutrição Josué de Castro - Universidade Federal do Rio de Janeiro, Rio de Janeiro, RJ.

Endereço para correspondência:

Gloria Valeria da Veiga

Universidade Federal do Rio de

Janeiro. Instituto de Nutrição Josué

de Castro

Av. Brigadeiro Trompowisky, s/no Ilha do Fundão, CCS, bloco J, $2^{\circ}$ andar, Ilha do Fundão.

21941-590 - Rio de Janeiro, RJ,

Brasil

E-mail: gvveiga@globo.com

\begin{abstract}
RESUMO
Introdução e objetivo: A grande preocupação com a aparência e a forma física e a constante pressão para manterem baixo peso corporal são fatores que levam a distorções da imagem corporal e tornam os bailarinos um grupo de risco para o desenvolvimento de transtornos alimentares (TA). O objetivo deste estudo foi avaliar a percepção da imagem corporal e sua associação com comportamentos de risco para TA em bailarinos profissionais. Métodos: Foram estudados 61 bailarinos (39 mulheres e 22 homens) de uma instituição representante da elite do balé clássico brasileiro. A avaliação dos comportamentos de risco para TA foi feita com base nas versões em português dos questionários Eating Attitudes Test (EAT-26) e Bulimic Investigatory Test Edinburgh (BITE). A Escala de Silhuetas de Stunkard foi utilizada para avaliação da percepção da imagem corporal. A associação entre as variáveis foi avaliada com base na razão de prevalência (RP) e seus respectivos intervalos de confiança (IC) de 95\%. Resultados: Trinta e um bailarinos (50,8\%) gostariam de ter a silhueta menor que a autopercebida como usual. A presença de comportamentos de risco para TA foi 2,71 vezes maior (IC 95\% = 1,02 - 7,18) entre os que desejavam ter silhueta menor que a usual e 2,64 vezes maior (IC 95\% = 1,20 - 5,80) entre aqueles que desejavam ter silhueta menor que a considerada mais saudável, quando comparados com os que estavam satisfeitos com a sua silhueta. Conclusão: A alta frequência de insatisfação com o corpo encontrada pode estar colaborando para ocorrência de comportamentos de risco para TA nos bailarinos investigados.
\end{abstract}

Palavras-chave: dança, anorexia nervosa, bulimia nervosa.

\begin{abstract}
Introduction and objective: Great concern with physical appearance and fitness as well as constant pressure in maintaining low body weight make ballet dancers a risk group for the development of eating disorders (ED). The objective of the present study was to evaluate the body image perception and its association with risk behavior for ED in professional ballet dancers. Method: Sixty-one dancers (39 women and 22 men) who represent the Brazilian classical ballet elite were assessed. The Portuguese versions of Eating Attitudes Test (EAT-26) and Bulimic Investigatory Test Edinburgh (BITE) questionnaires were used to evaluate de risk behavior for ED. Stunkard's standard silhouettes were used to assess body image perception. Association between variables was evaluated through the prevalence ratio (PR) and respective 95\% confidence interval (CI). Results: Thirty-one dancers (50.8\%) would like to have smaller silhouette than the usual. The presence of risk behavior for ED was 2.71 times higher ( $\mathrm{Cl}$ 95\%=1.02-7.18) among those who would like to have smaller silhouette than the usual and 2.64 times higher $(\mathrm{Cl} 95 \%=1.20-5.80)$ among those who would like to have smaller silhouette than the ones considered healthier. Conclusion: The high frequency of body dissatisfaction found can be collaborating for the onset of risk behavior for ED in professional ballet dancers.
\end{abstract}

Keyword: dancing, anorexia nervosa, bulimia nervosa.

\section{INTRODUÇÃO}

Bailarinos são um grupo de risco para o desenvolvimento de transtornos alimentares (TA) devido às rigorosas exigências de desempenho físico e estética corporal necessários para o exercício da profissã ${ }^{(1-5)}$. Essas exigências incluem valores de índice de massa corporal $\left(I M C=\right.$ peso/estatura $\left.{ }^{2}\right)$ e percentual de gordura corporal (\%GC) abaixo dos valores encontrados para a população em geral(2,6), levando à busca constante do "peso ideal" e contribuindo para a insatisfação com a imagem corporal ${ }^{(7,8)}$, uma vez que apenas a mi- noria dos bailarinos consegue se manter, naturalmente, dentro dos padrões exigidos.

A imagem corporal consiste no modo pelo qual o corpo se apresenta para cada indivíduo ${ }^{(9)}$. O processo de formação da imagem corporal pode ser influenciado por uma série de fatores, incluindo a relação com crenças e valores inseridos em uma cultura ${ }^{(10)}$, como no meio profissional dos bailarinos, onde o corpo magro e esguio é altamente valorizado. A percepção da imagem corporal pode ser mais importante na determinação de TA do que o próprio peso ${ }^{(11,12)}$. 
As complicações clínicas decorrentes dos $\mathrm{TA}^{(13)}$, além de comprometer a saúde, podem levar ao afastamento das atividades profissionais, uma vez que estas dependem de perfeita condição física.

O objetivo deste estudo foi avaliar a insatisfação com a imagem corporal e sua associação com a presença de comportamentos de risco para TA em bailarinos clássicos profissionais do Rio de Janeiro. Diante da escassez de trabalhos sobre o assunto no Brasil este estudo poderá trazer subsídios para a conscientização de bailarinos e instrutores quanto à importância de uma relação saudável com a imagem corporal para a prevenção de TA.

\section{MÉTODOS}

O estudo foi do tipo transversal, realizado em uma instituição representante da elite do balé clássico brasileiro. Entre os 79 bailarinos (50 mulheres e 29 homens) que exerciam a profissão na instituição, no período da coleta de dados, realizada de julho a setembro de 2006, cinco estavam em licença médica, duas estavam grávidas e um encontrava-se de licença para dançar no Exterior, sendo excluídos do estudo, totalizando 71 bailarinos elegíveis. Dez indivíduos não aceitaram participar da pesquisa, sendo o número final de participantes de 61 indivíduos (14,1\% de perda).

Para avaliar a presença de comportamentos de risco para TA foi utilizada a versão em português, traduzida e adaptada por Nunes et al.(14), do Eating Attitudes Test (EAT-26) e a versão em português desenvolvida por Cordás e Hochgraf(15) do Bulimic Investigatory Test Edinburgh (BITE). O resultado com base no EAT foi classificado como positivo (EAT+), classificando os indivíduos com presença de padrões alimentares anormais, quando era obtida pontuação maior que 20 e, com base no BITE (BITE+), classificando os indivíduos com presença de padrão alimentar não usual, quando era obtida pontuação maior ou igual a 10. Neste estudo foi considerada presença de comportamentos de risco para TA quando era obtido EAT e/ou BITE positivo.

Para avaliação da percepção da imagem corporal foi utilizada a Escala de Silhuetas de Stunkard ${ }^{(16)}$, onde são apresentadas nove silhuetas, para cada sexo, com diferentes tamanhos corporais, numeradas da menor (mais magra) para a maior (mais gorda). Essa escala foi considerada uma medida válida da imagem corporal em brasileiros, tendo boa correlação com o IMC e com a insatisfação corporal e também mostrou-se capaz de distinguir indivíduos com ou sem TA ${ }^{(17)}$. Os bailarinos foram solicitados a assinalar: 1) percepção da sua silhueta atual; 2) a silhueta que desejavam ter; e 3) a silhueta que julgavam como a mais saudável. Foram calculadas as diferenças entre o número da silhueta que representava a usual e o número da que foi referida como desejada (usual - desejada) e a diferença entre o número da silhueta considerada mais saudável e o número da referida como desejada (mais saudável - desejada). Foram então criadas as seguintes variáveis:

- Pontos de diferença entre a silhueta desejada e a autopercebida como silhueta usual (variável contínua).

- Silhueta desejada em relação à autopercebida como usual, categorizada em: igual, maior e menor.

- Silhueta desejada em relação à autopercebida como mais saudável, categorizada em: igual, maior e menor.

Aqueles que desejavam ter silhueta menor que a autopercebida como usual foram considerados insatisfeitos com sua imagem corporal. Devido à baixa frequência na categoria "maior", esta foi excluída na análise da associação com comportamentos de risco para TA.

Investigou-se a frequência da percepção da imagem corporal por sexo e faixa etária (abaixo ou acima da mediana: 35 anos).

Para cálculo do IMC aferiram-se peso com balança eletrônica com variação de $50 \mathrm{~g}$ e estatura por meio de estadiômetro com variação de 0,5cm, segundo técnica proposta por Gordon et al.(18) . A composição corporal foi estimada pela bioimpedância elétrica, sistema braço-perna. Para o cálculo da massa livre de gordura (MLG) foram utilizadas equações próprias para atletas ${ }^{(19,20)}$. O \%GC foi obtido por diferença.

Para caracterização dos bailarinos, investigaram-se, com base em questionário fechado e autopreenchido, as seguintes variáveis: tempo (em anos) como bailarino profissional; idade como contínua e categorizada em abaixo e igual ou acima da mediana de 35 anos, idade de início da prática de balé.

Para análise dos dados foi utilizado o software Statistical Package for Social Sciences (SPSS), versão 13.0. Foram calculadas as médias, os desvios-padrão e frequências das variáveis. Foi utilizado o teste do quiquadrado para comparar as frequências, o teste t de Student para comparar médias e valor de $p<0,05$ para significância estatística. A força da associação entre a presença de comportamentos de risco para TA e a insatisfação com a silhueta autopercebida como usual foi avaliada com base na razão de prevalência (RP) e seus respectivos intervalos de confiança (IC) de 95\%. Estimaram-se ainda as odds ratio (OR) bruta e ajustada pelas variáveis sexo, faixa etária e IMC.

O estudo foi aprovado pelo Comitê de Ética em Pesquisa do Núcleo de Estudos de Saúde Coletiva (Nesc) da Universidade Federal do Rio de Janeiro. Antes do início da coleta de dados, todos os participantes leram e assinaram o Termo de Consentimento Livre e Esclarecido, onde objetivos e procedimentos da pesquisa eram informados.

\section{RESULTADOS}

Entre os bailarinos investigados, 22 eram homens (36,0\%) e 39 (64,0\%), mulheres. A idade média foi de $36,8 \pm 10,5$ anos e a de início da prática de balé, de 6,9 2,6 anos para as mulheres e 16,6 3,7 anos para os homens $(p<0,001)$. As mulheres apresentaram média de \%GC maior do que os homens (18,2 $\pm 4,8 \%$ vs 9,7 $\pm 1,56 \%, p<0,001)$; os homens apresentaram maiores médias de IMC $\left(22,7 \pm 1,7 \mathrm{~kg} / \mathrm{m}^{2}\right.$ vs 19,4 $\left.\pm 1,6 \mathrm{~kg} / \mathrm{m}^{2}, p<0,001\right)$ e MLG $(61,8 \pm 4,1 \mathrm{~kg}$ vs 41,8 $\pm 4,0 \mathrm{~kg}, \mathrm{p}<0,001)$.

Não houve diferença entre homens e mulheres quanto às médias de pontos obtidos no EAT e no BITE e nem quanto às classificações nos dois questionários; 11,5\% dos avaliados apresentaram "padrões alimentares anormais" (EAT+) e 29,5\%, "padrão alimentar não usual" (BITE+); duas mulheres (3,3\%) obtiveram pontuação $\geq 20$ no BITE, o que caracteriza a presença de "comportamento alimentar compulsivo". Trinta e um por cento apresentaram presença de comportamentos de risco para TA (resultado positivo em, pelo menos, um dos dois questionários) sem diferença entre homens e mulheres (tabela 1).

Tabela 1. Médias e desvios-padrão (DP) da pontuação no Eating Attitudes Test (EAT) e no Bulimic Investigatory Test Edinburgh (BITE) e frequência de comportamentos de risco para transtornos alimentares (TA) em bailarinos clássicos profissionais segundo sexo.

\begin{tabular}{l|c|c|c|c|c|c|c}
\hline \multirow{2}{*}{ Variáveis } & \multicolumn{2}{c|}{ Total } & \multicolumn{2}{c|}{$\begin{array}{c}\text { Homens } \\
(\mathbf{n = 2 2})\end{array}$} & \multicolumn{2}{c|}{$\begin{array}{c}\text { Mulheres } \\
\text { (n=39) }\end{array}$} & \multicolumn{1}{c}{$\begin{array}{c}\text { Valor } \\
\text { de } \mathbf{p}^{1}\end{array}$} \\
\cline { 2 - 8 } & Média & DP & Média & DP & Média & DP & \\
\hline Pontuação no EAT & 12,0 & 8,1 & 11,4 & 8,0 & 12,4 & 8,2 & 0,647 \\
\hline Pontuação no BITE & 7,4 & 5,4 & 7,2 & 4,1 & 7,5 & 6,0 & 0,830 \\
\hline $\begin{array}{l}\text { Presença de padrões ali- } \\
\text { mentares anormais (EAT+) }\end{array}$ & 7 & 11,5 & 3 & 13,6 & 4 & 10,3 & 0,691 \\
\hline $\begin{array}{l}\text { Presença de padrão ali- } \\
\text { mentar não usual (BITE+) }\end{array}$ & 18 & 29,5 & 6 & 27,3 & 12 & 30,8 & 0,774 \\
\hline $\begin{array}{l}\text { Presença de comporta- } \\
\text { mentos de risco para TA } \\
\text { (EAT+ ou BITE+) }\end{array}$ & 19 & 31,1 & 7 & 31,8 & 12 & 30,8 & 0,932 \\
\hline
\end{tabular}

Teste t de Student.

${ }^{2}$ Teste do qui-quadrado. 
Para 95,0\% dos bailarinos, a silhueta autopercebida como usual e a considerada mais saudável está entre de no 1 e 4; 75,4\% ( $n=46)$ apontaram as silhuetas de no 1 ou 2 como as desejadas; entretanto, maior proporção de mulheres desejava ter silhueta no 1 ou 2 (92,3\% 모 $45,5 \%, p<0,001$ ), enquanto maior proporção de homens pretendia ter as silhuetas de $\mathrm{n}-3$ ou 4 (54,5\% vs 7,7\%, p < 0,001) (tabela 2).

Tabela 2. Distribuição das silhuetas autopercebidas pelos bailarinos como usual, mais saudável e desejada, segundo o sexo.

\begin{tabular}{|c|c|c|c|c|c|c|}
\hline \multicolumn{7}{|c|}{ HOMENS ( $n=22$ ) } \\
\hline \multirow[t]{2}{*}{ Silhueta escolhida' } & \multicolumn{2}{|c|}{ Usual } & \multicolumn{2}{|c|}{ Mais saudável } & \multicolumn{2}{|c|}{ Desejada } \\
\hline & $\mathrm{n}$ & $\%$ & $\mathrm{n}$ & $\%$ & $\mathrm{n}$ & $\%$ \\
\hline 1 ou 2 & 7 & 31,8 & 8 & 36,4 & 10 & 45,5 \\
\hline 3 ou 4 & 14 & 63,6 & 13 & 59,1 & 12 & 54,5 \\
\hline$\geq 5$ & 1 & 4,5 & 1 & 4,5 & 0 & 0 \\
\hline \multicolumn{7}{|c|}{ MULHERES $(n=39)$} \\
\hline \multirow[t]{2}{*}{ Silhueta escolhida' } & \multicolumn{2}{|c|}{ Usual } & \multicolumn{2}{|c|}{ Mais saudável } & \multicolumn{2}{|c|}{ Desejada } \\
\hline & $\mathrm{n}$ & $\%$ & $\mathrm{n}$ & $\%$ & $\mathrm{n}$ & $\%$ \\
\hline 1 ou 2 & 22 & 56,4 & 24 & 61,5 & 36 & 92,3 \\
\hline 3 ou 4 & 15 & 38,5 & 15 & 38,5 & 3 & 7,7 \\
\hline$\geq 5$ & 2 & 5,1 & 0 & 0 & 0 & 0 \\
\hline \multicolumn{7}{|l|}{ Valor de $p^{2}$} \\
\hline homens vs mulheres & \multicolumn{2}{|c|}{0,159} & \multicolumn{2}{|c|}{0,092} & \multicolumn{2}{|c|}{$<0,001^{3}$} \\
\hline
\end{tabular}

Metade dos bailarinos (50,8\%) gostaria de ter a silhueta menor que a autopercebida como usual, independente do sexo e faixa etária, e mais de 1/3 (36,1 \%) gostariam de ter silhueta menor do que a considerada mais saudável. Esse desejo era quase duas vezes maior entre as mulheres e mais frequente entre os mais jovens (54,8\% vs 16,7\%, $p=0,002$ ), enquanto que a ambição de ter a mesma silhueta que a considerada mais saudável foi mais frequente entre os mais velhos (80,0\% vs 41,9\%, p =0,002) (tabela 3).

A presença de comportamentos de risco para TA foi 2,7 vezes maior $(p=0,026)$ entre os que desejavam ter silhueta menor que a usual e 2,6 vezes maior $(p=0,012)$ entre aqueles que almejavam ter silhueta menor que a considerada mais saudável, quando comparados com os que não apresentavam insatisfação com a imagem corporal (tabela 4). Pela análise com base na odds ratio, a associação se manteve após ajuste pelas variáveis sexo, faixa etária e IMC (OR bruta = 4,1; IC 95\%= 1,1-14,8; OR ajustada = 5,96; IC 95\% = 1,2-28,5 para os que desejavam ter silhueta menor que a autopercebida como usual e OR =4,2; IC 95\% =1,3-3,8; OR ajustada = 4,5; IC 95\% = 1,1-7,7 para os que preferiam ter silhueta menor que a autopercebida como mais saudável).

\section{DISCUSSÃO}

Segundo Alvarenga(21) o corpo tornou-se objeto de consumo e a magreza sinônimo de beleza, valor e realização. A imagem que o indivíduo cria de seu corpo é muitas vezes, para ele, mais legítima que o próprio corpo, tendo importante participação na determinação de comportamentos de risco para $\mathrm{TA}^{(12)}$

Os bailarinos, principalmente as mulheres, também estão expostos à pressão social do culto à magreza; entretanto, o padrão físico imposto pela profissão é ainda mais rígido. Ao se alcançar o grau de bailarino profissional é necessário manter o alto nível da técnica e a constante
Tabela 3. Frequências das silhuetas desejadas em relação às silhuetas autopercebidas como usual e mais saudável por bailarinos clássicos profissionais segundo o sexo e faixa etária.

\begin{tabular}{|c|c|c|c|c|}
\hline Variáveis & $\begin{array}{l}\text { Total } \\
\text { n (\%) }\end{array}$ & $\begin{array}{c}\text { Homens } \\
(\mathrm{n}=22) \\
n(\%)\end{array}$ & $\begin{array}{c}\text { Mulheres } \\
(\mathrm{n}=39) \\
\mathrm{n}(\%)\end{array}$ & Valor de $p^{1}$ \\
\hline
\end{tabular}

Silhueta desejada em relação à silhueta autopercebida como usual ${ }^{2}$

\begin{tabular}{l|c|c|c|c}
\hline Igual & $24(39,3)$ & $11(50,0)$ & $13(33,3)$ & \\
\hline Menor & $31(50,8)$ & $8(36,4)$ & $23(59,0)$ & 0,233 \\
\hline Maior & $6(9,8)$ & $3(13,6)$ & $3(7,7)$ & \\
\hline
\end{tabular}

Silhueta desejada em relação à silhueta autopercebida como mais saudável ${ }^{2}$

\begin{tabular}{l|c|c|c|c}
\hline Igual & $37(60,7)$ & $15(68,2)$ & $22(56,4)$ & \\
\hline Menor & $22(36,1)$ & $5(22,7)$ & $17(43,6)$ & 0,062 \\
\hline Maior & $2(3,3)$ & $2(9,1)$ & 0 & \\
\hline Variáveis & $\begin{array}{c}\geq \mathbf{3 5} \text { anos } \\
(\mathbf{n = 3 0 )} \\
\mathbf{n}(\%)\end{array}$ & $\begin{array}{c}<\mathbf{3 5} \text { anos } \\
(\mathbf{n}=\mathbf{3 1}) \\
\mathbf{n}(\%)\end{array}$ & Valor de $\mathbf{p}^{\mathbf{1}}$ & \\
\hline
\end{tabular}

Silhueta desejada em relação à silhueta autopercebida como usual ${ }^{2}$

\begin{tabular}{l|c|c|c|c}
\hline Igual & & $12(40,0)$ & $12(38,7)$ & \\
\hline Menor & & $17(56,7)$ & $14(45,2)$ & 0,230 \\
\hline Maior & & $1(3,3)$ & $5(16,1)$ & \\
\hline
\end{tabular}

Silhueta desejada em relação à silhueta autopercebida como mais saudável ${ }^{2}$

\begin{tabular}{|c|c|c|c|}
\hline Igual & $24(80,0)$ & $13(41,9)$ & \\
\hline Menor & $5(16,7)$ & $17(54,8)$ & $0,007^{3}$ \\
\hline Maior & $1(3,3)$ & $1(3,2)$ & \\
\hline
\end{tabular}

Tabela 4. Razão de prevalência (RP) e intervalos de confiança (IC) de 95\% para presença de comportamentos de risco para transtornos alimentares segundo silhuetas desejadas em bailarinos clássicos profissionais.

\begin{tabular}{c|c|c|c|c|c|c}
\hline Variáveis & $\mathrm{N}$ & $\mathrm{n}$ & $\%$ & $\mathrm{RP}$ & IC 95\% & Valor de $\mathrm{p}^{1}$ \\
\hline
\end{tabular}

Silhueta desejada em relação à silhueta autopercebida como usual ${ }^{2}$

\begin{tabular}{c|c|c|c|c|c|c}
\hline Igual & 24 & 4 & 16,7 & 1,00 & & \\
\hline Menor & 31 & 14 & 45,2 & 2,71 & $1,02-7,18$ & 0,026 \\
\hline
\end{tabular}

Silhueta desejada em relação à silhueta autopercebida como mais saudável ${ }^{2}$

\begin{tabular}{c|c|c|c|c|c|c}
\hline Igual & 37 & 7 & 18,9 & 1,00 & & \\
\hline Menor & 22 & 11 & 50,0 & 2,64 & $1,20-5,80$ & 0,012 \\
\hline
\end{tabular}

Teste do qui-quadrado.

Usada Escala de Silhuetas de Stunkard.

melhora do desempenho. A manutenção de excelente qualidade física torna-se a necessidade básica dos profissionais desse gabarito, que precisam, incansavelmente, equilibrar preparo físico, força, leveza e expressões dramáticas ${ }^{(22)}$. Frente a tantas exigências físicas, os bailarinos profissionais podem se sentir insatisfeitos com seus corpos, buscando métodos inadequados para alcançar aquilo que desejam, prejudicando sua saúde e carreira.

No presente estudo, metade dos bailarinos gostaria de ter um corpo mais magro. A parcela significante de indivíduos, principalmente entre os mais jovens e entre as mulheres, que desejava ter silhueta menor do que aquela considerada mais saudável indica que o desejo de ter silhueta menor do que a usual, provavelmente, não está ligado a questões de saúde e sim de estética. Entre pacientes com 
anorexia nervosa, o desejo de ter silhueta menor que a considerada mais saudável está ligado à própria doença(17); entretanto, entre os bailarinos, as exigências físicas da profissão podem estar impulsionando esse desejo.

As silhuetas mais escolhidas como desejadas (silhuetas $n \circ-1$ e 2) no presente trabalho foram menores do que as observadas em estudo realizado no Brasil com 186 praticantes de caminhada ${ }^{(10)}$ e em estudo realizado com gêmeos e seus familiares nos Estados Unidos(23). Nesses estudos também foi utilizada a Escala de Silhuetas de Stunkard(16) para avaliar a imagem corporal e observou-se que as silhuetas no 3 e 4 foram as mais desejadas por mulheres e homens, respectivamente. Esses resultados reforçam que o padrão corporal magro exigido para bailarinos de ambos os sexos pode ter um efeito ainda maior na insatisfação corporal dos mesmos.

No presente estudo, a silhueta no 4 foi escolhida por apenas 11,5\% (27,3\% dos homens e 2,6\% das mulheres) dos bailarinos como a desejada e nenhum indivíduo escolheu silhueta superior à $\mathrm{n}$ ㄴ 4, que foi estabelecida como ponte de corte para magreza no estudo de Bulik et al. ${ }^{(23)}$, mostrando que todos os bailarinos estudados desejavam silhuetas correspondentes à magreza.

A insatisfação com o próprio corpo pode ser um fator precipitante dos TA, contribuindo, juntamente com outros fatores etiológicos, para o seu início ${ }^{(24)}$. No presente estudo, a frequência de comportamentos de risco para TA foi mais que o dobro entre os que estavam insatisfeitos e desejavam silhueta menor quando comparados com os que se sentiam satisfeitos com a imagem corporal. Esses achados concordam com estudo realizado com 513 mulheres de 12 a 19 anos de Porto Alegre, onde se observou que, entre as mulheres com IMC normal, mas que se sentiam gordas, 66\% apresentavam comportamento alimentar de risco ou anormal, enquanto que, entre as que se sentiam magras, apenas 14,8\% apresentavam esse tipo de comportamento, mostrando como a percepção do corpo pode ser mais influente que o próprio peso na ocorrência de comportamentos de risco para $\mathrm{TA}^{(12)}$.

\section{REFERÊNCIAS}

1. Melin P, Araújo AM. Transtornos alimentares em homens: um desafio diagnóstico. Rev Bras Psiquiatr 2002;24:73-6.

2. Janout $V$, Janoutová G. Eating disorders risk groups in the Czech Republic: cross-sectional epidemiologic pilot study. Biomed Papers 2004;148:189-93.

3. Sudi K, Öttl K, Payerl D, Baumgartl P, Tauschmann K, Müller W. Anorexia Athletica. Nutrition 2004;20:657-61.

4. Sundgot-Borgen J, Torstveit MK. Prevalence of eating disorders in elite athletes is higher than in the general population. Clin J Sport Med 2004;14:25-32.

5. Thomas JJ, Keel PK, Heatherton TF. Disordered eating attitudes and behaviors in ballet students: examination of environmental and individual risk factors. Int J Eat Disord 2005;38:263-8.

6. Koutedakis Y, Jamurtas A. The dancer as a performing athlete. Sports Med 2004;34:651-61.

7. Smith AR, Joiner TE. Examining body image discrepancies and perceived weight status in adult Japanese women. Eat Behav 2008;9:513-5.

8. Nilsson K, Sundbom E, Hägglöf B. A longitudinal study of perfectionism in adolescent onset anorexia nervosa-restricting type. Eur Eat Disord Rev 2008;16:386-94.

9. Saikali CJ, Soubhia CS, Scalfo BM, Cordás A. Imagem corporal nos transtornos alimentares. Rev Psiquiatr Clin 2004;31:164-6.

10. Damasceno VO, Lima JRP, Vianna JM, Vianna VRA, Novaes JS. Tipo físico ideal e satisfação com a imagem corporal de praticantes de caminhada. Rev Bras Med Esporte 2005;11:181-6.

11. Lautenbacher S, Thomas A, Roscher S, Strian F, Pirke KM, Krieg JC. Body size perception and body satisfaction in restrained and unrestrained eaters. Behav Res Ther 1992;30:243-50.

12. Nunes MA, Olinto MTA, Barros FC, Camey S. Influência da percepção do peso e do índice de massa corporal nos comportamentos alimentares anormais. Rev Bras Psiquiatr 2001;23:21-27.

13. Assunção CL, Cabral MD. Complicações clínicas da anorexia nervosa e bulimia nervosa. Rev Bras Psiquiatr 2002;24:29-33.

14. Nunes MA, Bagatini LF, Abuchaim AL, Kunz A, Ramos D, Silva JA, et al. Distúrbios da conduta alimentar: considerações sobre o Teste de Atitudes Alimentares (EAT). Revista ABP-APAL 1994;6:7-10.

15. Cordás TA, Hochgraf PO. O "BITE": instrumento para avaliação da bulimia nervosa - versão para o português. J Bras Psiquiatr 1993;42:141-4
Damasceno et al. ${ }^{(10)}$ observaram que o tipo físico considerado ideal pelas mulheres é aquele com 20,5\% de GC e IMC de 20,0 kg $/ \mathrm{m}^{2}$ e, para os homens, aquele com 9,8\% de GC e IMC de 23,1 kg/ $\mathrm{m}^{2}$. Esses valores encontram-se acima das médias encontradas para os bailarinos deste estudo que, apesar disso, se mostram insatisfeitos com seu corpo, indicando o quanto o tipo físico almejado por eles é bastante rigoroso. As médias de IMC encontradas no presente estudo, para homens e mulheres, apresentam-se dentro da faixa de eutrofia proposta pela Organização Mundial de Saúde ${ }^{(25)}$. As médias de \%GC encontram-se abaixo da faixa de normalidade proposta por Bray et al. ${ }^{(26)}$; entretanto, estão dentro da média esperada para bailarinos ${ }^{(27)}$.

Em tempos de culto à magreza é comum que os indivíduos, mesmo eutróficos, se sintam insatisfeitos com seus corpos que não estão dentro desse padrão de beleza tão severo ${ }^{(10,12,23,28-30)}$. Entretanto, essa alta frequência de insatisfação entre os bailarinos é preocupante, uma vez que estes já possuem corpos bastante magros e o desejo de perder ainda mais peso pode estimular a prática de comportamentos de risco para TA.

Pode-se concluir que, mesmo possuindo corpos magros, com baixo \%GC, muitos bailarinos estão insatisfeitos com seu corpo e desejam ser mais magros devido ao rígido padrão estético exigido nessa profissão. Considerando, então, a profissão de bailarino como importante fator de risco para ocorrência de TA, a prevenção desse problema torna-se ainda mais difícil, já que o desejo de se manter na profissão é, para muitos, mais forte que o desejo de ser saudável.

Uma possível limitação do estudo é o número reduzido de bailarinos avaliados, embora tenham sido investigados cerca de $85 \%$ dos profissionais que compõem o corpo de baile oficial da instituição. Mais estudos em outras instituições que agreguem dançarinos de elite são necessários para conhecer as peculiaridades e riscos para os transtornos alimentares nesses indivíduos, de modo a tornar as ações preventivas mais eficientes.

Todos os autores declararam não haver qualquer potencial conflito de interesses referente a este artigo.
16. Stunkard A, Sorensen T, Schulsinger F. Use of the Danish Adoption Register for the study of obesity and thinness. In: Kety et al, editors. The genetics of neurological and psychiatric disorders. Raven Press: New York; 1983.

17. Scagliusi FB, Alvarenga M, Polacow VO, Cordas TA, Queiroz GKO, Coelho D et al. Concurrent and discriminant validity of the Stunkard's figure rating scale adapted into Portuguese. Appetite 2006;47:77-82.

18. Gordon CC, Chumlea WC, Roche AF. Stature, Recumbent length and weight. In: Lohman TG, Roche AF, Martorell R. Anthropometric standardization reference manual. Illinois: Human Kinetics Books; 1988.

19. Houtkooper LB, Going SB, Westfall CH, Lohman TG. Prediction of fat-free body corrected for bone mass from impedance and anthropometry in adult females. Med Sci Sports Exerc 1989;21:39.

20. Oppliger RA, Nielsen DH, Hoegh JE, Vance CG. Bioelectrical impedance prediction of fat-free mass for high school wrestlers validated. Med Sci Sports Exerc 1991;23:73.

21. Alvarenga M. A mudança na alimentação e no corpo ao longo do tempo. In: Philippi ST, Alvarenga, M, editores. Transtornos alimentares: uma visão nutricional. Barueri: Manole; 2004. p. 2-20

22. Leal M. A preparação física na dança. Rio de Janeiro: Sprint; 1998.

23. Bulik CM, Wade TD, Heath AC, Martin NG, Stunkard AJ, Eaves $\sqcup$. Relating body mass index to figural stimuli: population-based normative data for Caucasians. Int J Obes Relat Metab Disord. 2001; 25:1517-24.

24. Hetherington MM. Eating disorders: diagnosis, etiology, and prevention. Nutrition 2000;16(7):547-51.

25. World Health Organization Expert Committee. Physical status: the use and interpretation of anthropometry. WHO Technical Report Series, no. 854. Geneva: WHO; 1995. p.263-344.

26. Bray GA, Bouchard C, James WPT. Definitions and proposed current classification of obesity. In: Bray GA, Bouchard C, James WPT, editors. Handbook of obesity. New York: Marcel Dekker; 1998. p. 25.

27. Heyward VH, Stolarczyk LM. Avaliação da composição corporal aplicada. Barueri: Manole; 2000.

28. Fiates GMR, Salles RK. Fatores de risco para o desenvolvimento de distúrbios alimentares: um estudo em universitárias. Rev Nutr Campinas 2001;14:3-6.

29. Kakeshita IS, Almeida SS. Relação entre o índice de massa corporal e a percepção da auto-imagem em universitários. Rev Saúde Pública 2006;40:497-504.

30. Nicolaou M, Doak C, Dam R, Hosper K, Seidell J, Stronks K. Body size preference and body weight perception among two migrant groups of non-Western origin. Public Health Nutr 2008;11:1332-41. 